\section{Emergency medicine training}

$\mathrm{Sir}$, it is essential that emergency medicine hospital staff have a basic understanding of dental and maxillofacial pathology. We analysed the local trauma data at Derriford Hospital from March-May 2020 using the emergency department software. Searches in treatment summaries were used to identify cases where dental and oral and maxillofacial surgery trainees were requested to give input to a patient's care.

We then designed a training package that covered the in-demand areas and the most prevalent referrals. This consisted of a lecture with various visual aids based around anatomy, diagnosis and treatment delivery. This was delivered on several occasions, both in person and remotely, to clinicians of various seniorities within the emergency department from consultants to nurses. The training was enthusiastically received on all occasions as evidenced in subsequent feedback.

We feel that the targeted training package has addressed many of the deficits and there is significant room within the emergency medicine training programme to measure, focus and develop such content to the needs of junior doctors. ${ }^{1}$

We hope to encourage other dental and oral and maxillofacial surgery departments to get involved in training within their respective emergency departments and recognise that this can bring benefits in the form of improved co-operation, reduced referral load for minor conditions and better patient care.

M. Bosov, K. Skorko, Plymouth, UK

\section{Reference}

1. The Royal College of Emergency Medicine. Curriculum and Assessment Systems for Training in Emergency Medicine. August 2015 Curriculum. Approved 23 November 2015. Available at: https://www.rcem.ac.uk/ docs/Training/RCEM 2015 Curriculum Applicable from_August_2016_approved_23_Nov_2015_ATCF RTT_DRE-EM_additions_July2017\%20-\%20JCST\%20 amendments.pdf (accessed 23 February 2021).

https://doi.org/10.1038/s41415-021-2798-1

\section{Dental education}

\section{Dental teacher training}

Sir, historically healthcare accepted that knowledge and experience translated to competent teaching, but the disparity can be vast. Medicine recognised the professional obligation for all to teach, subsequently adopting it as a core role for doctors. Similarly, dental educators acknowledged the need for teacher training (TT), but the profession persists with limited guidance and no mandate for it. Would dentistry benefit from mandatory TT, or would the barriers to implementation overshadow progress?

A sophisticated student body has emerged following the increase in tuition fees and rise in graduate entry standards, culminating in a shift towards higher teaching expectations. The literature has long documented concerns from students and educators over teaching standards in hospitals, demonstrating desire to change. The positive impact regular TT has on student learning is recognised and highlighted by employees' requests for teacher experience. Collectively, this illustrates that a system-wide desire and argument for TT exists, suggesting that no change to current practice could be detrimental to the profession.

Possible solutions addressing shortfalls in TT have been identified in the literature, bestowing responsibility on clinicians, institutions and regulatory bodies alike. Favourable outcomes have been reported with the integration of specific programmes, increased funding, protecting teaching time and modifying attitudes towards teaching.

Despite recognition from all parties of

TT's positive impact, a number of challenges would limit mandatory implementation. All solutions demand increased time and economic input, in an era where there is an ever-growing pressure to reduce costs, a downward trend of teachers compared to students and an increase in patient numbers, it seems unrealistic that significant changes could be made soon. Furthermore, reliable methods of measuring the impact of TT remain elusive and there is conflicting literature on the long-term effectiveness of teaching qualifications. This creates uncertainty for institutions in justifying budgetary allocations to this domain.

The presumption that clinical expertise alone can fulfil dental teachers' educational obligation is no longer tenable and the benefits of formal TT are understood. The conflicting demands of clinics, teaching and administration, economic and time constraints create a complex situation. Further research is needed to conclude mandatory TT's overall impact, but input from the dental regulatory bodies could be a step in the right direction.

H. Hawrot, Leeds, UK https://doi.org/10.1038/s41415-021-2799-0

\section{Infection control}

\section{PW-I for all? Not yet}

Sir, I read with interest the recent paper on the recommendations to use povidone iodine mouthwash as part of routine infection prevention and control (IP\&C) measures in dental practice. ${ }^{1}$

There are several limitations in the methodology of this study that should be considered when assessing this recommendation. The manuscript implies that the British and European Standard BS EN $14476^{2}$ was fully complied with in testing this disinfectant; this was not the case, for example:

- Concentration tested: BS EN 14476 specifies that products can only be tested at a concentration of $80 \%(97 \%$, with a modified method for special cases)

- Controls used: The standard recommends using a well characterised disinfectant (such as formaldehyde) as a control of the test system. This was not used. A negative control comprising the mouthwash diluent ie iodine as the active ingredient removed, is a more appropriate control than water

- Neutralisation effectiveness evaluation: The authors highlight quite rightly the importance of disinfectant neutralisation to ensure '...that there were no after effects of the test product beyond the specified contact time.' However, no validation of this method was presented, invalidating the authors' results

- Activity in the presence of soiling: To be fair the authors did use concentrations specified in EN 14476, however, these are not relevant to the oral cavity. The standard specifies that 'the interfering substance shall be chosen according to the conditions of use laid down for the product.' A relevant contaminant for an oral rinse product is mucin.

Finally, the concept of a pre-procedural anti-viral rinse as part of IP\&C protocols has recently been considered by a number of groups ${ }^{3}$ and insufficient evidence has been noted to recommend its routine use, principally, the washout effect of saliva would negate any potential anti-viral effect shortly after application unless the active agent demonstrated substantivity. The use of an anti-viral mouthwash as an IP\&C measure is an interesting concept but the data from this 